\title{
Thromboembolism as a Cause of Renal Artery Occlusion and Acute Kidney Injury: The Recovery of Kidney Function after Two Weeks
}

\author{
Niina Koivuviita ${ }^{a} \quad$ Risto Tertti $^{a} \quad$ Maija Heiro $^{a} \quad$ Ilkka Manner $^{b}$ \\ Kaj Metsärinne \\ aDivision of Nephrology, Department of Medicine, and ${ }^{\mathrm{b}}$ Department of Radiology, \\ Turku University Hospital, Turku, Finland
}

\section{Key Words}

Acute kidney injury · Angioplasty · Renal artery $\cdot$ Thromboembolism

\section{Abstract}

Thromboembolic occlusion is a rare cause of acute kidney injury (AKI). It may lead to permanent loss of renal function. Our patient, who had dilated cardiomyopathy and prosthetic aortic valve, presented with AKI due to thromboembolic arterial occlusion of a solitary functioning kidney. After 2 weeks delay, local intra-arterial thrombolytic treatment with recombinant tissue plasminogen activator was performed without sufficient effect. However, a subsequent percutaneous transluminal angioplasty with stenting was successful. Diuresis began immediately, and renal function was fully recovered after 2 weeks. Although there had been no evident arterial circulation in the kidney, we think that minor flow through subtotal occlusion of the main renal artery made the hibernation of kidney tissue possible and contributed to the recovery. Thus, even after prolonged ischemia, revascularization can be useful.

(C) 2014 S. Karger AG, Basel

\section{Background}

Acute renal arterial occlusion (RAO) because of thromboembolism is a rare event. The clinical presentation of RAO is variable, depending on whether the embolic occlusion affects both renal arteries or the artery of a solitary kidney, or occurs in a single kidney with a 
contralateral functioning kidney. It has been pointed out that the more prompt the diagnosis and the treatment, the better the renal outcome. However, the literature is rather limited.

We present a patient with thromboembolic RAO of a solitary kidney. Due to the difficulty in making the clinical diagnosis, the endovascular treatment was done only after 14 days from the beginning of the symptoms. Still, almost total recovery of renal function was achieved after the procedure.

Our aim is to increase the awareness of renal artery embolism as a possible cause of acute kidney injury (AKI) and to encourage performance of radiological interventions even if diagnostic procedures are delayed.

\section{Case Presentation}

A 61-year-old man presented to our hospital with AKI. His medical history consisted of dilated cardiomyopathy and operative correction of both aortic and mitral valves. The aortic valve had been replaced with prosthesis. He had congenital aplasia of the right kidney, and thus, a solitary kidney on the left side. Two years prior to this event, the estimated glomerular filtration rate was normal. The following medications were used: losartan (50 mg/day), spironolactone $(25 \mathrm{mg} /$ day), and warfarin. The international normalized ratio had been suboptimal (1.4) about 4 weeks before arriving to the hospital.

The patient was admitted to the hospital because of a severe left-sided, colicky abdominal pain and total anuria, which had begun about $12 \mathrm{~h}$ before. Clinical status revealed minor abdominal tenderness on the left side. There were no clinical findings suggesting abdominal catastrophe such as perforation or occlusion.

The patient's blood pressure was stable at approximately 140/80 mm Hg. Electrocardiogram showed sinus rhythm (approx. 75 beats $/ \mathrm{min}$ ) and left bundle branch block configuration as earlier. Plasma creatinine was elevated at $384 \mu \mathrm{mol} / \mathrm{l}$ and the potassium level was 5.2 $\mathrm{mmol} / \mathrm{l}$. No acidosis was noted. In urine samples, hematuria, proteinuria and pyuria were detected but bacterial cultures of urine were negative.

Ultrasound of the left kidney did not show any obvious abnormalities. Abdominal computed tomography (CT) without contrast media was performed because of the clinical suspicion of ureteral calculus causing colicky pain and AKI. However, the only finding was a wedge-shaped hypodensity in the spleen, suggestive of infarction. We observed a decline in the glomerular filtration rate and hemodialysis treatment was started on the second day after admission.

During the next several days, anuria continued. C-reactive protein values started to rise (up to $348 \mathrm{mg} / \mathrm{l}$ ). A transthoracic and esophageal echocardiography was normal. The blood cultures were negative. There were no signs of pneumonia in the chest radiograph. After 13 days, a Doppler ultrasound of the left kidney revealed absence of blood flow in the renal artery. A subtotal thrombotic occlusion of the renal artery was detected by angiography (fig. 1). It was presumed to be of embolic origin and treated with intra-arterial tissue plasminogen activator thrombolysis resulting only in a minor reduction of intraluminal thrombus. Subsequent percutaneous transluminal angioplasty (PTA) and stenting were successfully performed with restoration of blood flow to the kidney. Diuresis appeared immediately and hemodialysis treatment could be stopped after 2 weeks. At present, plasma creatinine has stabilized at the level of $170-180 \mu \mathrm{mol} / \mathrm{l}$. 


\section{Discussion}

Here, we presented a case of renal artery thromboembolism as a cause of AKI. The time after the onset of symptoms to the revascularization of renal artery was long (14 days). It is remarkable that kidney function recovered almost entirely.

AKI as defined by the RIFLE criteria, is observed in nearly $8 \%$ of hospitalized patients, and in more than $50 \%$ of patients in the intensive care unit [1]. Approximately $70-75 \%$ of AKI cases in the hospital are due to prerenal disease and acute tubular necrosis [2]. Renal artery embolism is an infrequent but important cause of AKI. It is detected in only $1 \%$ of AKI cases [2]. The major causes of renal embolic disease are thromboemboli (systemic clot emboli) and atheroemboli (cholesterol emboli). In situ thrombosis of a stenotic renal artery is a less common cause of renal infarction [3]. In $94 \%$ of patients, systemic emboli commonly originate from the heart. It is typically seen in patients with atrial fibrillation. The reported incidence of renal thromboembolism in patients with atrial fibrillation was $2 \%$ in a series of almost 30,000 patients followed for up to 13 years [4]. Other significant cardiac disorders include ischemic heart disease, cardiomyopathy and valvular disease as in our case $[5,6]$. Furthermore, valvular vegetations in infective endocarditis, rarely tumor, and paradoxical embolism through a patent foramen ovale are potential embolic sources.

Most patients experience acute onset of abdominal, flank, or back pain. The pain is typically constant and without significant modifying factors [7]. The symptoms of acute embolic renal infarction may mimic a pain similar to renal colic, and is often misdiagnosed as a renal calculus because of similar presenting symptoms. The presence of hematuria does not differentiate renal calculus from acute renal infarction. Reported frequency of hematuria in renal infarction varies widely from 45 to $74 \%$ [5-7]. However, hematuria may be considerably delayed because development of structural damage takes several hours. Thus, an initial lack of hematuria does not rule out acute renal infarction. The differential diagnosis should also include pyelonephritis, and other causes of acute, nontraumatic abdominal pain such as appendicitis, cholecystitis, small-bowel obstruction, perforated peptic ulcer, pancreatitis, and diverticulitis. Our patient had hematuria on admission, but also pyuria, reported to occur in $15 \%$ of renal infarction patients [7], supporting differential diagnosis of pyelonephritis. However, the urine bacterial culture was negative.

The clinical manifestations of renal artery thromboembolism vary substantially, depending on the amount of renal mass behind the embolus. If the embolism is bilateral or blocking the artery of a solitary kidney it can lead to total anuria as in our case. In the two largest case series of acute renal embolism, the mean serum creatinine value on admission was $115 \pm 27$ [7] and $158 \pm 147 \mu \mathrm{mol} / \mathrm{l}$ [6]. In the first mentioned study, only 4 out of 20 (20\%) patients had renal function impairment on admission, whereas in the study by Hazanov et al. [6] nearly $60 \%$ of patients $(24 / 38)$ had renal failure, and $8 \%(3 / 38)$ of them were dialysis-dependent. However, in 9 renal failure patients, the kidney function recovered as a result of treatment.

According to the literature, clinical diagnosis of acute renal infarction is usually based on typical, though unspecific, clinical findings and a history suggestive of increased risk for thromboembolism. At this point, hematuria and elevated serum lactate dehydrogenase (LDH) suggest the possible diagnosis of acute renal infarction. Serum LDH is a characteristic marker for cell necrosis and is known to be elevated in patients with acute renal infarction. The mean serum LDH levels on hospital admission in case series of renal infarction patients were from 412 to $812 \mathrm{U} / \mathrm{l}[5,7]$. Unfortunately, we had no LDH value of our patient. However, little is known about the time after pain onset when LDH elevation occurs. In the study by Hazanov et al. [6], serum LDH was taken from 20 patients on admission. The LDH 
was normal in 5 and increased on the next day. In 17 patients studied by Domanovits et al. [5], the LDH increased from 412 to $932 \mathrm{U} / \mathrm{l}$ (mean) during the next $24 \mathrm{~h}$. Since serum LDH is not specific for acute renal infarction, other causes, such as acute myocardial infarction, mesenteric embolism, and hemolysis must be excluded. Measurement of urinary LDH may be helpful [8]. This enzyme is too large to be filtered and therefore its excretion rate is normal in extrarenal disorders. In comparison, in renal infarction and renal transplant rejection, there is an increase in the excretion rate directly from the kidneys.

Spiral CT without contrast agent is usually the preferred initial test for flank pain, being the gold standard for the diagnosis of kidney and ureteral stones, which are much more common than renal infarction [6]. If there is no evidence of stone disease on unenhanced spiral CT, an enhanced CT scan should be performed to assess for renal infarction. However, the risk of contrast nephropathy, although in most cases self-remitting, has to be taken into account especially when estimated glomerular filtration rate is below $30 \mathrm{ml} / \mathrm{min}$. As these patients can have a remarkably decreased glomerular filtration rate, Doppler ultrasound would probably be the methodological choice of a clinician. 'No-flow signal' as a consequence of total occlusion of renal artery is obviously easy to detect as in our patient. However, the sensitivity of these imaging procedures was addressed in a series of 44 patients with atrial fibrillation and a diagnosis of embolic renal infarction. The sensitivity was $97 \%(36 / 37)$ with radioisotope renal scan, $80 \%(12 / 15)$ with contrast-enhanced CT, and only $11 \%(3 / 27)$ with renal ultrasound [6]. This probably reflects the difficulties to detect small emboli in peripheral renal artery branches.

The time to diagnosis following presentation is often more than 2 days, with fewer than $50 \%$ of cases being diagnosed promptly [6]. The human kidney tolerates complete ischemia for about 60-90 min at normothermia [9]. In dogs, renal ischemia for $2 \mathrm{~h}$ was followed by gradual recovery of renal function over 2-3 weeks. Ischemia for $4 \mathrm{~h}$ caused irreversible renal damage [10]. However, there are case reports about recovery of kidney function even after weeks of total occlusion [11]. Successful recovery of renal function after such prolonged periods of occlusion can be explained by the presence of collateral vessels that maintain kidney viability or by the incomplete occlusion of the renal artery. The kidneys receive collateral blood flow from lumbar, suprarenal, and ureteral vessels that communicate either prerenally into the hilum or intrarenally via perforating arterioles of the capsule [12]. In our patient, angiography revealed no obvious collaterals but a very thin residual flow channel in the main renal artery (fig. 1). Blood flow to the kidneys is normally about $20 \%$ of the cardiac output or $1,000 \mathrm{ml} / \mathrm{min}$. The blood supply to the kidneys is one of the highest in the body since only $10 \%$ of the delivered oxygen is sufficient to satisfy the renal metabolic demands. Thus, it is possible that even severely compromised perfusion is capable of keeping kidney tissue viable and hibernating, although there is no filtration, and the patient is totally anuric.

Therapeutic guidelines for the treatment of renal artery embolism have not been established, and because of the rarity of the disease, it is unlikely that the superiority of a particular therapy will be evaluated in prospective randomized clinical trials. The diagnosis is essential especially in patients with total parenchymal involvement secondary to bilateral emboli or to a unilateral main renal artery embolus in a solitary kidney, since thrombolysis, anticoagulation, or embolectomy may minimize the loss of renal function. The maximum duration of complete RAO beyond which thrombolysis would no longer be beneficial is not known. One study reported little benefit after several hours [13], while others noted some benefit up to several days later [14]. Later therapy is likely to be effective only with incomplete occlusion. In our case, treatment with thrombolysis for $5 \mathrm{~h}$ was ineffective while PTA with stenting still restored the kidney function. This demonstrates the possible advantage of angioplasty treatment even after an extended period of time from the start of 
the symptoms. In an experimental renal artery stenosis model, it has been shown that structural microvascular (vessels between 0 and $200 \mu \mathrm{m}$ ) rarefaction can be detected already 6 weeks after renal artery stenosis induction, and that functional changes precede the structural ones [15]. It seems likely that the severity of microvascular damage and loss may be the link between reversible and irreversible renal injury. In the future, we would need noninvasive methods to assess the intrarenal microvascular architecture, distribution and function.

\section{Conclusion}

Even after prolonged anuria and apparent lack of renal circulation, restoration of renal function may be seen after revascularization by thrombolysis, PTA and stenting. Renal artery thromboembolism should be suspected and recognized in AKI of unknown etiology, especially in patients with solitary kidneys, and predisposing disorders.

\section{Disclosure Statement}

The authors have no conflicts of interest to disclose.

\section{References}

1 Hoste E, Clermont G, Kersten A, Venkataraman R, Angus D, De Bacquer D, Kellum J: Rifle criteria for acute kidney injury are associated with hospital mortality in critically ill patients: a cohort analysis. Crit Care 2006;10:R73.

2 Liaño F, Pascual J: Epidemiology of acute renal failure: a prospective, multicenter, community-based study. Madrid Acute Renal Failure Study Group. Kidney Int 1996;50:811-818.

-3 Paris B, Bobrie G, Rossignol P, Le Coz S, Chedid A, Plouin P: Blood pressure and renal outcomes in patients with kidney infarction and hypertension. J Hypertens 2006;24:1649-1654.

-4 Frost L, Engholm G, Johnsen S, Møller H, Henneberg E, Husted S: Incident thromboembolism in the aorta and the renal, mesenteric, pelvic, and extremity arteries after discharge from the hospital with a diagnosis of atrial fibrillation. Arch Intern Med 2001;161:272-276.

-5 Domanovits H, Paulis M, Nikfardjam M, Meron G, Kürkciyan I, Bankier A, Laggner A: Acute renal infarction. Clinical characteristics of 17 patients. Medicine (Baltimore) 1999;78:386-394.

6 Hazanov N, Somin M, Attali M, Beilinson N, Thaler M, Mouallem M, Maor Y, Zaks N, Malnick S: Acute renal embolism. Forty-four cases of renal infarction in patients with atrial fibrillation. Medicine (Baltimore) 2004;83:292-299.

7 Huang CC, Lo H, Huang HH, Kao WF, Yen DH, Wang LM, Huang CI, Lee CH: ED presentations of acute renal infarction. Am J Emerg Med 2007;25:164-169.

8 London I, Hoffsten P, Perkoff G, Pennington T: Renal infarction. Elevation of serum and urinary lactic dehydrogenase (LDH). Arch Intern Med 1968;121:87-90.

9 Semb C: Partial resection of the kidney: anatomical, physiological and clinical aspects. Ann R Coll Surg Engl 1956;19:137-155.

10 Hamilton P, Phillips R, Hiller A: Duration of renal ischemia required to produce uremia. Am J Physiol 1948;152:517-522.

-11 Zankl AR, Dengler TJ, Andrassy M, Volz HC, Katus HA, Zeier M: Recovery of renal function after delayed percutaneous dilatation of a subtotal in-stent restenosis of the renal artery in a left solitary kidney. NDT Plus 2009;2:236-238.

12 Abrams H, Cornell S: Patterns of collateral flow in renal ischemia. Radiology 1965;84:1001-1012. Blum U, Billmann P, Krause T, Gabelmann A, Keller E, Moser E, Langer M: Effect of local low-dose thrombolysis on clinical outcome in acute embolic renal artery occlusion. Radiology 1993;189:549-554.

14 Siablis D, Liatsikos E, Goumenos D, Karnabatidis D, Voudoukis T, Barbalias G, Vlahogiannis J: Percutaneous rheolytic thrombectomy for treatment of acute renal-artery thrombosis. J Endourol 2005;19:68-71.

-15 Iliescu R, Fernandez S, Kelsen S, Maric C, Chade A: Role of renal microcirculation in experimental renovascular disease. Nephrol Dial Transplant 2010;25:1079-1087. 
Case Reports in

Nephrology and

Urology
Case Rep Nephrol Urol 2014;4:82-87

DOI: $10.1159 / 000362538$

Koivuviita et al.: Thromboembolism as a Cause of Renal Artery Occlusion and Acute

Kidney Injury: The Recovery of Kidney Function after Two Weeks

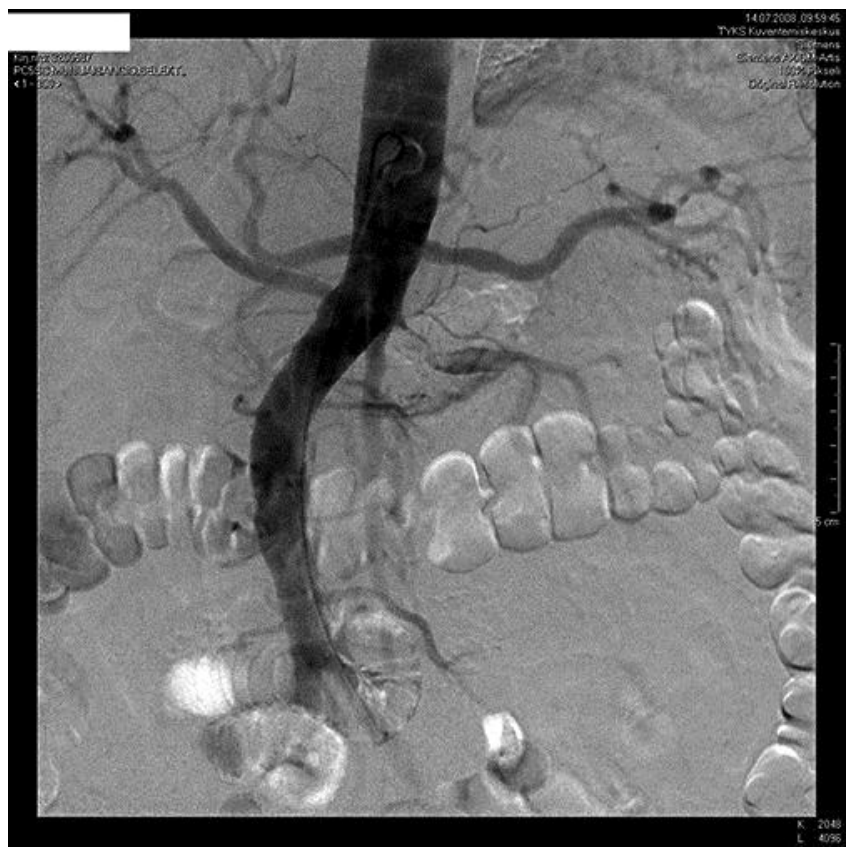

Fig. 1. Renal artery angiogram before thrombolysis and angioplasty. 